\title{
REVIEWS
}

\section{Viral myocarditis-diagnosis, treatment options, and current controversies}

\author{
Ari Pollack, Amy R. Kontorovich, Valentin Fuster and G. William Dec
}

\begin{abstract}
Myocarditis—a frequent cause of dilated cardiomyopathy and sudden cardiac death—typically results from cardiotropic viral infection followed by active inflammatory destruction of the myocardium. Characterization of this disease has been hampered by its heterogeneous clinical presentations and diverse aetiologies. Advances in cardiac MRI and molecular detection of viruses by endomyocardial biopsy have improved our ability to diagnose and understand the pathophysiological mechanisms of this elusive disease. However, therapeutic options are currently limited for both the acute and chronic phases of myocarditis. Several randomized, controlled trials have demonstrated potential benefit with immunosuppressive and immunomodulatory therapies, but further investigations are warranted. In this Review, we explore the pathophysiology, natural history, and modes of diagnosis of myocarditis, as well as evidence-based treatment strategies. As novel imaging techniques and human in vitro models of the disease emerge, the landscape of therapies for myocarditis is poised to improve.
\end{abstract}

Pollack, A. et al. Nat. Rev. Cardiol. 12, 670-680 (2015); published online 21 July 2015; doi:10.1038/nrcardio.2015.108

\section{Introduction}

According to the current WHO classification of cardiomyopathies, myocarditis is clinically and pathologically defined as an inflammatory disease of the myocardium diagnosed by established histological, immunological, and immunohistochemical criteria. ${ }^{1,2}$ Myocarditis often results from common viral infections that have a predilection towards entry into the myocardium. Animal models of viral myocarditis predict a maladaptive post-viral immune-mediated response, which leads to eventual myocardial cell dysfunction and compromised contractility. Although the pathogenesis is not well characterized in humans, modern advances in PCR technology have enabled detection of enteroviruses, adenoviruses, parvovirus B19, and human herpesvirus 6 in patients with acute myocarditis. ${ }^{3,4}$ Several other aetiologies of myocarditis have also been implicated, including HIV, Chagas disease, toxins, medications, and autoimmune phenomena (Figure 1). ${ }^{1,5}$ Clinically, myocarditis can manifest as acute heart failure, ventricular arrhythmias, or cardiogenic shock, and is associated with substantial morbidity and mortality. ${ }^{5}$ Children diagnosed with acute myocarditis have only a $60 \%$ likelihood of transplantation-free survival at 10 years. ${ }^{6}$ Myocarditis has been linked as the cause of sudden cardiac death in young adults in up to $12 \%$ of cases, and identified as the cause of dilated cardiomyopathy in $9 \%$ of patients. ${ }^{7-9}$

In medical practice, physicians rely on a combination of clinical features, laboratory analyses, and imaging

Competing interests

The authors declare no competing interests. findings to diagnose myocarditis. However, a definitive diagnosis relies on endomyocardial biopsy (EMB), an approach supported by the WHO as well as scientific statements provided by the AHA and ESC..$^{1,210}$ Despite a clear definition and the well-established morbidity and mortality associated with the disease, many patients with clinical manifestations of myocarditis do not undergo EMB, the current gold standard for diagnosis. Considering these established standards, it is unclear why such heterogeneity exists relating to the evaluation, diagnosis, and treatment of patients with myocarditis.

Perhaps the many aetiologies linked to the disease, along with its varied clinical presentations, hampers patient identification and prevents true consensus and acceptance of the current diagnostic standards. The development of the Dallas criteria, ${ }^{11}$ which rely on traditional histological stains, was an initial attempt at standardizing diagnostic guidelines. These criteria define myocarditis as either active or borderline, depending on the degree of inflammation and myocyte injury. ${ }^{11}$ However, legitimate concerns exist about the diagnostic accuracy and sensitivity of the Dallas criteria, which might be explained by the focal and transient nature of the inflammatory process. ${ }^{12-14}$ As a result, EMB with immunohistochemistry is gaining increasing acceptance in the diagnosis of myocarditis. ${ }^{15}$ Questions remain, however, about the need for routine biopsy in cases of suspected myocarditis, especially in light of the considerable advances in imaging technology with cardiac MRI (CMR).

Currently, aside from standard pharmacological treatment for patients with symptoms of heart failure and 


\section{Key points}

- Myocarditis is a frequent cause of dilated cardiomyopathy with heterogeneous clinical presentations and a wide range of clinical outcomes

- After infection by a cardiotropic virus, a maladaptive post-viral response ensues, which can cause myocardial cell dysfunction and compromised contractility

- Advances in our diagnostic capabilities using cardiac MRI as well as molecular detection of viruses by endomyocardial biopsy have improved our understanding and ability to characterize the disease

- Although comprehensive clinical recommendations specific to the treatment of myocarditis do not exist, modulation of the immune system is a promising therapeutic strategy as suggested by several randomized trials

left ventricular (LV) systolic dysfunction, comprehensive clinical practice guidelines specific to the treatment of myocarditis do not exist. For patients with cardiogenic shock due to acute fulminant myocarditis who deteriorate despite optimal medical therapy, mechanical circulatory support might be required as a bridge to recovery or heart transplantation..$^{15}$ Although specific types of autoimmune-related myocarditis, such as giantcell myocarditis and cardiac sarcoidosis, might respond to immunosuppressive therapy, tailored treatment strategies for viral myocarditis have yet to be established. Investigational treatment options, including immunosuppressive, immunomodulatory, anti-inflammatory, and antiviral agents, have been tested and reported in the literature with some promising results in specific patient populations. In this Review, we describe the pathophysiology, natural history, and techniques used for the diagnosis of myocarditis, as well as currently used and novel treatment strategies.

\section{Pathophysiology}

Myocarditis and the ensuing cardiomyopathy that might occur is caused by infectious and noninfectious agents (Figure 1). Over the past 4 decades, viral infection has increasingly been recognised as the most common aetiology. Using PCR technology and in situ hybridization techniques, viral RNA and DNA have been identified within the myocardium of affected patients. On an individual patient basis, causal associations with infection are difficult to make, owing to the invasive nature and low diagnostic yield of EMB. However, pathological and autopsy series have linked specific viral infections with up to $69 \%$ of cases of clinical myocarditis and/or pericarditis. ${ }^{16}$ Additionally, elevated serum levels of neutralizing antibody titres to viruses have been measured in patients with idiopathic cardiomyopathy. ${ }^{17}$ These findings further support the theory of a viral pathogenic basis for myocarditis-induced cardiomyopathy.

Overall, little is known about the early stages of acute viral myocarditis in humans, including viral entry and replication and cellular injury and death. Enteroviruses (most commonly coxsackie B viruses) are responsible for up to $25 \%$ of viral myocarditis cases ${ }^{16}$ and, of all the major pathogens, their mode of cardiac infectivity is the best characterized in animal models. Enteroviruses gain access to human hosts via the gastrointestinal or respiratory tracts; the heart is targeted secondarily. Infection of the myocardium by enteroviruses occurs in three phases (Figure 2): phase 1 involves viral entry into myocytes and activation of innate immunity; during phase 2 , viral replication and activation of acquired immune responses occur; and phase 3 is either resolution with recovery or development of dilated cardiomyopathy. In phase 1, viral entry is facilitated by decay accelerating factor and mediated through a specific cell-surface receptor, which allows internalization of the virus. Coxsackie B viruses and some adenoviruses exhibit tropism for cardiomyocytes via a common transmembrane receptor, the coxsackievirus and adenovirus receptor (CAR). ${ }^{18-20}$ When CAR is knocked out in rodent models, neither infection by coxsackieviruses nor histological evidence of myocarditis occur. ${ }^{21}$ Several days after the initial infection, persistent viral replication leads to myocyte necrosis, as first described in mice infected with coxsackievirus B3. ${ }^{22}$ Cellular degradation ensues (mediated by viral production of protease $2 \mathrm{~A}$ in the case of coxsackievirus $\mathrm{B} 3)_{,}{ }^{23}$ as does circulation of intracellular antigens, including myosin, which triggers the host's immune system. Natural killer cells, macrophages, and eventually
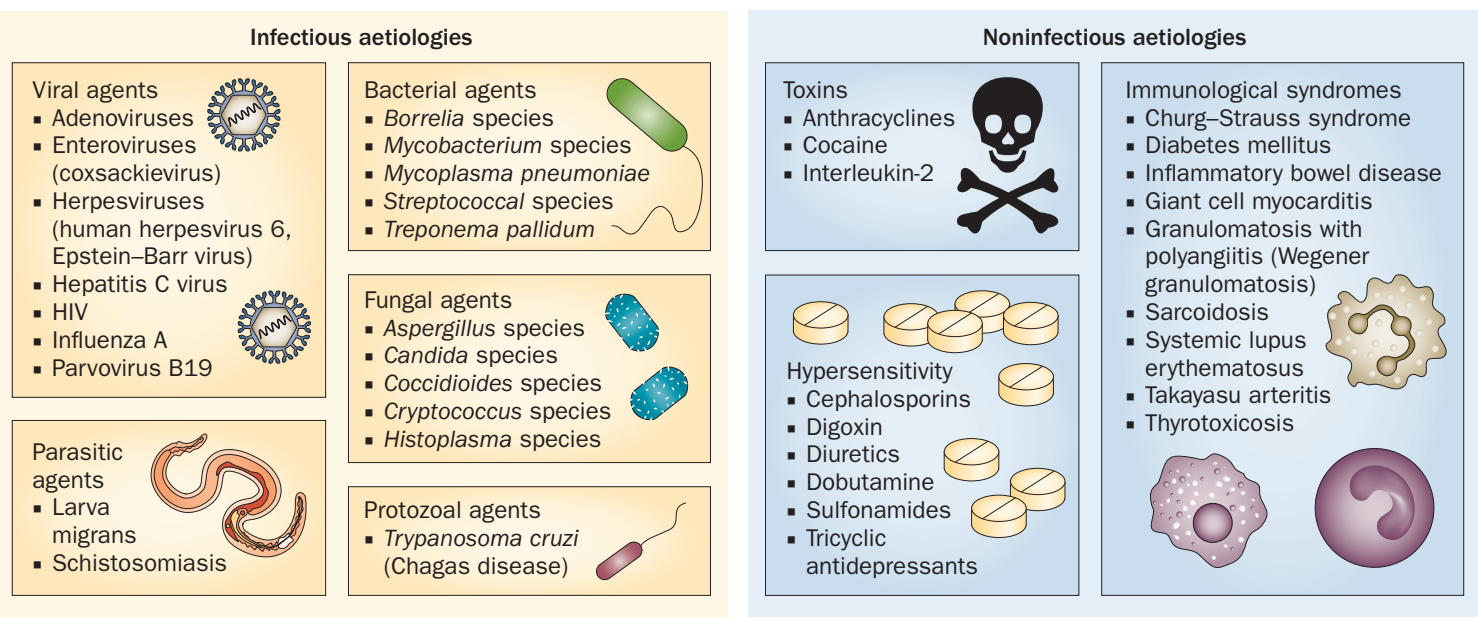

Figure 1 | Common causes of myocarditis. Viral infection is the most common aetiology, but several other aetiologies of myocarditis have also been implicated. 


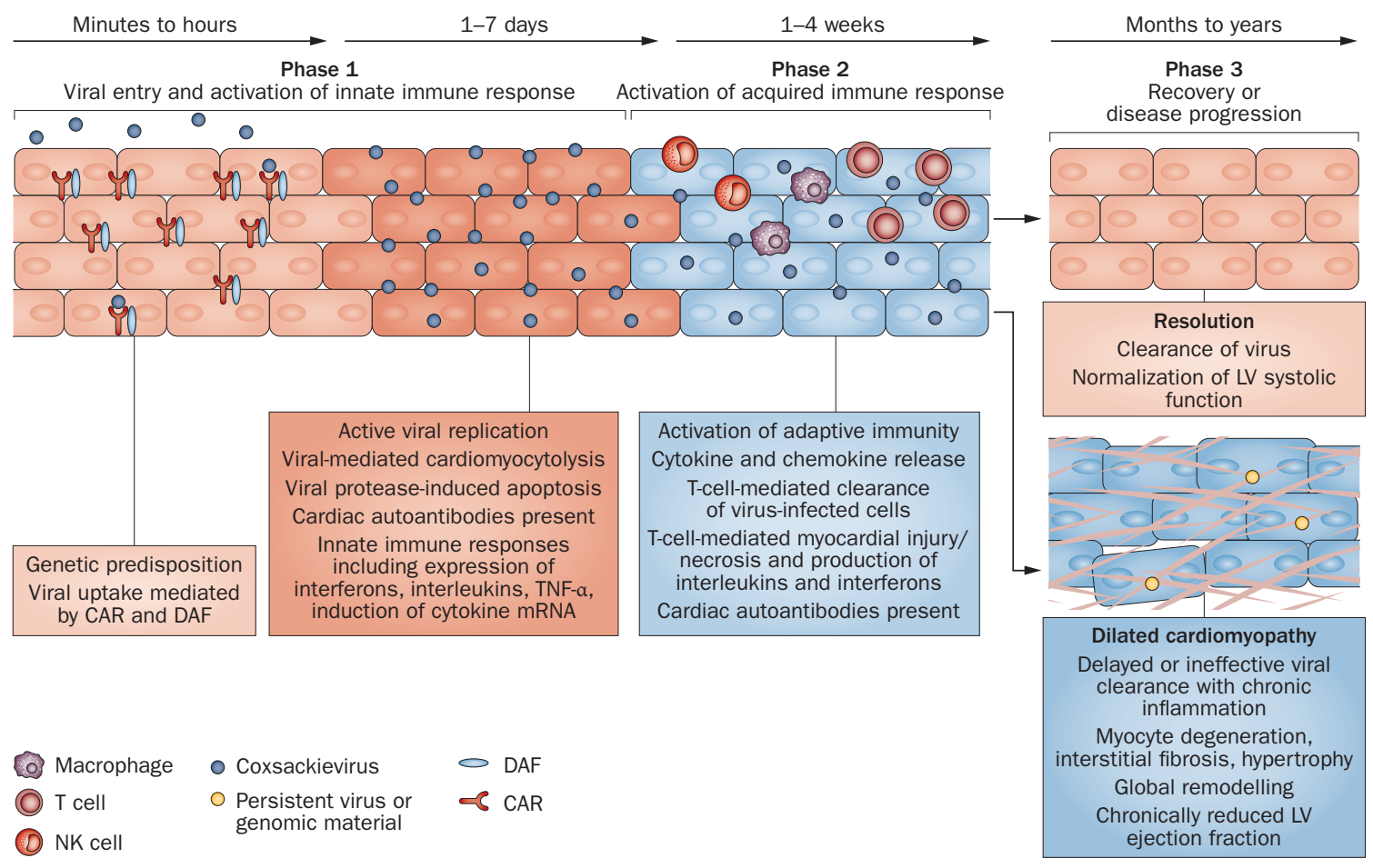

Figure 2 | Temporal phases of coxsackievirus-mediated myocarditis. Phase 1 commences with viral entry into the host and transit to the myocardium. In predisposed individuals, coxsackievirus binds to DAF on the cell surface, which shuttles the virus towards the CAR, localized at cell-cell junctions. The complex is internalized by the cell, triggering numerous cellular responses and activation of host innate immunity. Virus replication within the cardiomyocyte causes cell lysis, which prompts a set of cascades leading to the release of cytokines. In phase 2, both cellular and humoral responses contribute to autoimmune-mediated injury. The first immune cells recruited to the injured myocardium are NK cells, followed by macrophages. Infiltration of T cells typically peaks at 7-14 days; this timing coincides with the most severe phase of disease. As viral titres decrease, inflammation subsides (phase 3 ) and some individuals experience complete resolution of myocardial injury. However, in other patients, viral genomic material persists (in some cases up to 12 months; viral persistence throughout the entirety of end-stage disease until time of transplantation has been demonstrated), ${ }^{57}$ contributing to chronic inflammation and dilated cardiomyopathy. Abbreviations: CAR, coxsackievirus and adenovirus receptor; DAF, decay accelerating factor; LV, left ventricular; NK, natural killer; TNF, tumour necrosis factor.

T lymphocytes are recruited to the site of infection, causing further myocardial injury and marking the onset of phase 2. Autoimmune reactions-possibly facilitated by molecular mimicry-activate virus-specific $\mathrm{T}$ cells that target host myocardium. High levels of cytokines, particularly tumour necrosis factor (TNF), IL-1a, IL-1b, IL-2, and IFN- $\gamma$, are produced during this phase. These cytokines, together with antibodies to viral and cardiac proteins, can further potentiate cardiac damage and compromise systolic function through derangement of the contractile apparatus and/or interstitial cells and matrix proteins (for example dystrophin cleavage by protease $2 \mathrm{~A}$ ). The subacute phase of myocarditis (phase 2) can last weeks to several months and, in most patients, the immune response and ventricular contractile function improve as viral titres decrease. However, in some patients, the cardiomyopathy enters a chronic and irreversible phase. In summary, enteroviruses cause eventual systolic dysfunction through a cascade of cytotoxic events initiated by direct infection of the cardiomyocyte by the virus.

Other cardiotropic viruses can cause myocarditis by infection of cardiac endothelial cells. For example, parvovirus B19 can hibernate in bone marrow after primary infection in childhood; the virus later infects cardiac endothelium. Parvovirus B19 genomes have been found in endothelial cells of venuoles, small arteries, and arterioles in patients with fulminant myocarditis. ${ }^{24}$ In a series of EMBs, parvovirus B19 genomes were identified in $64 \%$ of patients with myocarditis compared with 7\% in normal controls. ${ }^{25}$ The virus exerts its pathogenic effects by activation of cytokines (IL- 6 and TNF) and induction of apoptosis, leading to endothelial dysfunction. If extensive enough, endothelial damage might compromise tissue perfusion, causing ischaemia and systolic dysfunction. This process is also marked by upregulation of adhesion molecules (for example E-selectin) on the endothelial cell surface, which home T lymphocytes to the myocardium..$^{24}$ Massive accumulation of lymphocytes in the coronary microcirculation can increase coronary resistance, trigger coronary vasospasm, and induce myocyte necrosis. Thus, although parvovirus does not directly infect cardiomyocytes, the downstream effects of endothelial cell infection can still lead to ventricular dysfunction and eventual cardiomyopathy. 
Although enteroviruses have historically been considered to be the most common cause of viral myocarditis, PCR data indicate that various viruses have a role in the aetiology of acute viral myocarditis. ${ }^{26}$ In a series of 172 patients with dilated cardiomyopathy, PCR demonstrated the following genomic distribution: parvovirus B19 (36.6\%), enteroviruses (32.6\%), human herpesvirus $6(10.5 \%)$, and adenoviruses $(8.1 \%) .{ }^{27}$ Whether this finding relates to evolutionary genomic changes in these viruses or geographical differences in viral prevalence and infectivity remains to be investigated.

\section{Presentation and natural history}

Myocarditis presents with heterogeneous signs and symptoms, ranging from subclinical disease to chest pain that can mimic myocardial infarction or pericarditis, refractory cardiogenic shock, or sudden cardiac death from ventricular fibrillation. ${ }^{28}$ Depending on the degree of LV dysfunction, acute and chronic heart failure might occur along with cardiac arrhythmias. A viral syndrome involving the respiratory or gastrointestinal tract with associated systemic symptoms might precede the onset of cardiac symptoms, although the occurrence of such a viral syndrome is highly variable. ${ }^{29}$ Serum cardiac biomarkers, specifically troponin I and troponin T, can be elevated in cases of myocarditis and help to confirm the diagnosis, but lack sensitivity. For example, in the Multicentre Myocarditis Treatment trial, the sensitivity of elevated troponin I levels in patients with biopsy-proven myocarditis was 34\%, specificity was $89 \%$, with a positive predictive value of $82 \% .{ }^{30}$ Troponin I and troponin $\mathrm{T}$ are more frequently elevated than creatinine kinase-MB, with increased levels of troponin I at presentation portending a worse prognosis. ${ }^{30}$ These findings have led to the routine measurement of cardiac biomarkers in patients with suspected myocarditis. Other serum markers of inflammation, including white blood cell count, erythrocyte sedimentation rate, and C-reactive protein levels, can be elevated in acute myocarditis, but are neither sensitive nor specific in terms of determining the presence or absence of active myocardial inflammation. ${ }^{30,31}$

Electrocardiogram findings are neither sensitive nor specific for the diagnosis of myocarditis; these abnormalities might include nonspecific ST-segment changes, T-wave inversions, and ST-segment elevations imitating acute myocardial ischaemia or infarction. ${ }^{32}$ Segmental wall motion abnormalities might be present, owing to the focal nature of the initial inflammatory process, and global echocardiographic wall motion abnormalities have also been reported. ${ }^{33}$ This finding is hardly surprising, because CMR studies of patients with myocarditis reveal an inflammatory process that evolves from focal to diffuse in the first few weeks after the clinical presentation. ${ }^{34}$ Accordingly, echocardiographic findings are largely dependent on both the manner and timing of a patient's presentation. Those patients with fulminant myocarditis typically have normal LV diastolic dimensions, but increased septal thickness at presentation, whereas patients with acute viral myocarditis might have either normal or increased diastolic dimensions, but normal septal thickness, consistent with other forms of acute dilated cardiomyopathy. ${ }^{35}$

The natural history of myocarditis is unpredictable. Acute myocarditis in patients who present with heart failure and mild LV systolic dysfunction typically recover in weeks to months. Among patients who present with significant LV dysfunction, $50 \%$ of patients will have complete resolution, $25 \%$ will go on to have chronic systolic dysfunction, and $25 \%$ will progress towards an end-stage cardiomyopathy resulting in transplantation or death. ${ }^{5,36}$ The prognosis of myocarditis is influenced by the specific aetiology together with particular diagnostic and clinical features at presentation. Mortality for biopsy-verified viral myocarditis has been reported as $20 \%$ and $56 \%$ at 1 year and 4.3 years, respectively. ${ }^{29}$ Patients with preserved LV function at diagnosis have a good prognosis, whereas patients with severe LV and biventricular dysfunction without haemodynamic collapse tend to do worse. Other clinical markers that might predict a poor prognosis include a history of syncope, a prolonged QRS duration, left bundle branch block, pulmonary hypertension, and NYHA class III or IV heart failure symptoms. ${ }^{15}$ Incorporating EMB findings, patients with NYHA functional class III or IV with positive immunohistology who are not receiving optimal heart failure therapy have the poorest prognosis, with a 5-year transplantation-free survival of only $39 \% .^{37}$ Interestingly, patients who presented with fulminant myocarditis and cardiogenic shock in an early series were reported to have an excellent long-term prognosis. ${ }^{38}$ However, in a subsequent observational study, 2 -year mortality was $>50 \% .{ }^{39}$ Patients with giantcell myocarditis have a substantially worse prognosis, with $<20 \%$ of patients surviving 5 years, but early initiation of immunosuppressive therapy or advanced mechanical support can influence the natural history of giant-cell myocarditis. ${ }^{40}$

\section{Diagnostic imaging}

CMR is a valuable noninvasive imaging technology that is especially useful for patients with myocarditis, by virtue of its capacity to detect inflammation, oedema, necrosis, and fibrosis within myocardial tissue. ${ }^{41}$ Several imaging sequences exist that can be used to differentiate and identify various characteristics associated with both acute and chronic myocarditis. ${ }^{42}$ T2-weighted imaging enables detection of myocardial oedema and tissue hyperaemia, which are features that are used as surrogates for myocardial inflammation. ${ }^{43}$ Contrast imaging with gadolinium enables both detection of early capillary leakage on the basis of T1-weighted early gadolinium enhancement, and accurate diagnosis of myocardial necrosis and fibrosis on the basis of late gadolinium enhancement (LGE) ${ }^{41,43}$ LGE is frequently observed in patients with active myocarditis in a pattern that is distinct from ischaemic myocardial injury. LGE imaging in myocarditis has indicated two common patterns of myocardial injury: either an intramural, rimlike pattern involving the septum, or a patchy epicardial 


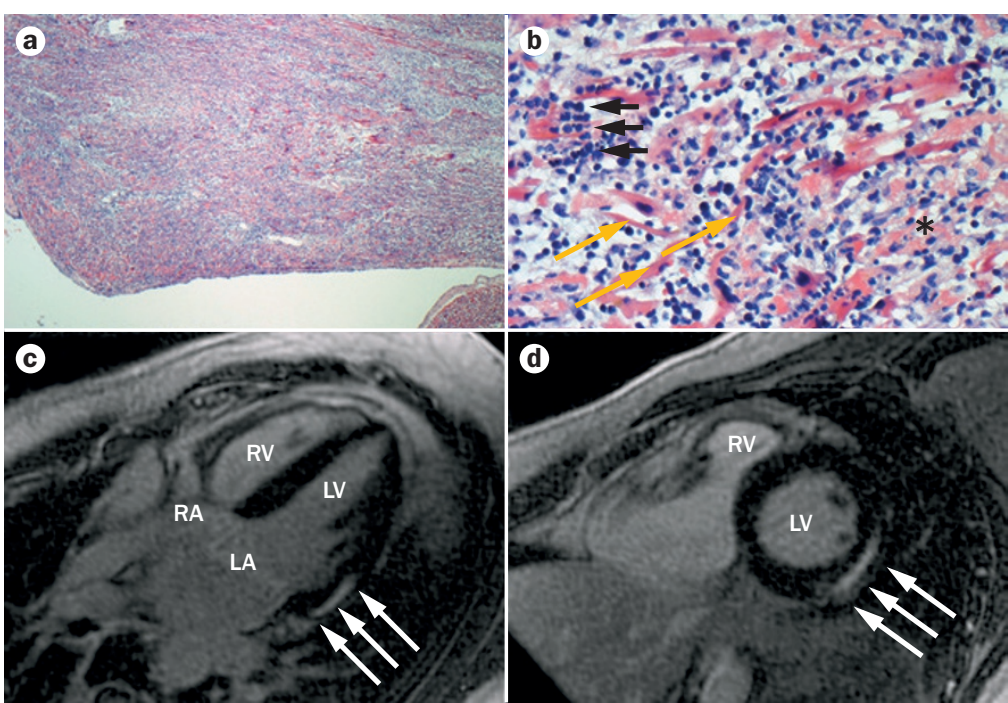

Figure 3 | Acute viral myocarditis is characterized by a destructive immune response in the myocardium. a | Substantial inflammatory infiltration (blue staining) is seen on haematoxylin and eosin staining of a left ventricular apical specimen from a woman aged 56 years with fulminant myocarditis (magnification $\times 100$ ). b | The same specimen shows profound lymphocyte and macrophage intrusion (black arrows), myocyte destruction (asterix), derangement of myocardial architecture (yellow arrows), and intracellular oedema (haematoxylin and eosin staining, magnification $\times 400$ ). $\mathbf{c} \mid$ Four-chamber and $\mathbf{d} \mid$ short-axis images acquired using cardiac MRI in a separate patient with acute myocarditis revealing abnormal patchy epicardial delayed gadolinium enhancement in the inferolateral segments. Abbreviations: LA, left atrium; LV, left ventricle; RA, right atrium; RV, right ventricle.

distribution involving the lateral free wall of the left ventricle (Figure 3). ${ }^{44}$ In a series of 222 consecutive patients with biopsy-proven myocarditis, the presence of LGE yielded a hazard ratio of 8.4 for all-cause mortality and 12.8 for cardiac mortality, independent of symptoms. ${ }^{45}$ A study has also shown that a decrease in the extent of LGE over time is often associated with an improvement in LV systolic function in patients with a subepicardial LGE pattern in the LV free wall. ${ }^{44}$ By contrast, patients whose intramural septal LGE pattern does not regress over time tend to have poor outcomes. ${ }^{44}$ Although excellent diagnostic correlation exists between CMR and EMB in troponin-positive patients without coronary artery disease who present with features consistent with myocarditis, diagnosis is more challenging with LGE in patients with less inflammation and a prolonged duration of symptoms. ${ }^{46}$

The ESC position statement on diagnosis of myocarditis affirms the use of CMR in clinically stable patients suspected of having myocarditis before EMB. ${ }^{1}$ A combination of CMR techniques is recommended, including T1-weighted early gadolinium enhancement, LGE, and T2-weighted oedema imaging, which indicates, at the tissue level, the presence of oedema, hyperaemia, and irreversible cell injury. This combined method has been termed the Lake Louise criteria. ${ }^{1,42}$ Contemporary CMR techniques used to characterize myocardial tissue enable the noninvasive diagnosis of myocarditis with high specificity, but less-than-ideal sensitivity. The presence of two of the three CMR characteristics listed in the Lake Louise criteria leads to a sensitivity of $67 \%$, specificity of $91 \%$, positive predictive value of $91 \%$, and negative predictive value of $69 \%$ for the diagnosis of myocarditis. ${ }^{42,43,47}$ The sensitivity of CMR for the diagnosis of myocarditis is likely to be limited in patients with mild myocardial inflammation, owing to inadequate spatial resolution; studies have shown that the diagnosis is made more frequently by CMR in patients with active myocarditis than in those with borderline myocarditis. ${ }^{46}$ Other important limitations associated with the diagnosis of myocarditis using only CMR is the absence of detailed information about the degree of inflammation provided by EMB and the inability to identify specific forms of myocarditis (viral, bacterial, giant cell, or eosinophilic) that require distinct therapeutic treatment. ${ }^{41,48}$

Although CMR is a promising technology, its sensitivity for the diagnosis of myocarditis must be improved, and additional imaging techniques should be explored for the acquisition of more-detailed information regarding both the mechanism and aetiology of the myocardial inflammation. One potential focus for research is in the development of novel imaging agents that highlight areas of active myocarditis on CMR. The detection of immune cell infiltration in experimental viral myocarditis by ${ }^{19} \mathrm{~F} \mathrm{MRI}$ in vivo is plausible with the use of biochemically inert perfluorocarbons. These perfluorocarbons are taken up by circulating macrophages, recruited to areas of myocardial infection and/or inflammation, and can be visualized by CMR. ${ }^{49}$ An alternative approach is to develop molecular labels for CMR-based detection of virus. Gadolinium-conjugated monoclonal antibodies are currently being studied for noninvasive molecular imaging of melanoma, and antibody-labelled superparamagnetic iron oxide can localize prostate cancer cells under MRI visualization. ${ }^{50,51}$ Development of such agents directed against antigens in the viral capsid might theoretically enable a noninvasive and robust global myocardial 'biopsy'.

The potential role of PET cardiac imaging for diagnosing myocarditis is intriguing. ${ }^{18} \mathrm{~F}$-fluoro-2-deoxyglucose PET ( $\left.{ }^{18} \mathrm{~F}-\mathrm{FDG}-\mathrm{PET}\right)$ is a molecular imaging technique that is highly sensitive to metabolically active processes, including inflammation. ${ }^{52}$ Although it has yet to be investigated in patients with viral myocarditis, studies have demonstrated the promising potential of ${ }^{18} \mathrm{~F}-\mathrm{FDG}$-PET in the diagnosis and evaluation of patients with cardiac sarcoidosis via the detection of active myocardial inflammation. ${ }^{53-55}$

\section{Role of EMB as a diagnostic tool}

EMB, using standardized histopathological (Dallas criteria $^{11}$ ) and immunohistochemical diagnostic criteria, is the current gold standard by which a diagnosis of myocarditis is made. The Dallas criteria define active myocarditis as an inflammatory infiltrate of the myocardium with necrosis and/or degeneration of adjacent myocytes not typical of the ischaemic damage associated with coronary artery disease (Figure 3). ${ }^{11}$ The infiltrates are usually lymphocytic, but might be neutrophilic or, occasionally, 
eosinophilic, and almost always include macrophages. 'Borderline myocarditis' is the term used when the inflammatory infiltrate is too sparse or myocyte injury is not demonstrated. ${ }^{11}$ The Dallas criteria are limited, however, by virtue of a high degree of interobserver variability in pathological interpretation and the inability to detect noncellular inflammatory processes, and yields diagnostic information in only $10-20 \%$ of patients. ${ }^{5,15}$ Therefore, immunohistochemistry with the use of a large panel of monoclonal and polyclonal antibodies is now obligatory to differentiate the inflammatory components present and the immunological processes activated. ${ }^{1}$ According to the WHO definition, active myocarditis is present with immunohistochemical detection of focal or diffuse mononuclear infiltrates ( $T$ lymphocytes and macrophages) using a cut-off of $>14$ cells per $\mathrm{mm}^{2}$, in addition to increased expression of HLA class II molecules. ${ }^{2}$ Molecular detection of viral genomic sequences in diseased myocardium is also feasible and, when coupled with immunohistochemical analysis, increases the diagnostic accuracy of EMB in addition to providing an aetiology and offering prognostic information. ${ }^{56-58}$ Whereas the Dallas criteria ${ }^{11}$ are not an accurate predictor of poor outcomes, immunohistological evidence of inflammatory infiltrates within the myocardium is associated with an increased risk of cardiovascular death and need for heart transplantation. ${ }^{37}$ Furthermore, when 120 patients with viral myocarditis were prospectively evaluated and divided into two groups on the basis of the absence or presence of enteroviral genomes in EMB samples, mortality and progression to end-stage cardiomyopathy was significantly greater in those with residual viral genome in the myocardium than in those with no virus detected..$^{59}$ Importantly, persistence of viral genome on repeat EMB was subsequently associated with progressive LV dysfunction, whereas spontaneous viral clearance was associated with improvement in systolic function. ${ }^{27}$ Information about the safety of particular treatments can also be gleaned from data obtained via EMB. Detection of specific HLA markers on EMB tissue sections combined with the absence of infectious agents (PCR-negative for viral genome) suggests either primary or postinfectious immune-mediated myocarditis, at which point immunosuppression might be considered. ${ }^{60}$

EMB performed early in the clinical presentation typically generates the most diagnostic information. However, EMB seems to be infrequently used to make the diagnosis, irrespective of time course. In 2007, the AHA/ACC/ESC consensus statement on the role of EMB in the management of cardiovascular disease provided 14 clinical scenarios in which the utility and safety of EMB were weighed against the risks of performing the procedure. ${ }^{48} \mathrm{~A}$ class I recommendation for early EMB is reserved for patients with rapidly advancing cardiomyopathy refractory to conventional therapy, or unexplained cardiomyopathy associated with ventricular arrhythmias or conduction disease.$^{48}$ However, these recommendations were based on application of the Dallas criteria, in which the diagnostic, prognostic, and therapeutic value is limited. ${ }^{37}$ The incorporation of immunohistochemical analysis for the detection of inflammation, as well as viral genome analysis, has improved the diagnostic and prognostic accuracy and usefulness of EMB. ${ }^{37,60}$ As a result, the 2013 ESC position statement on myocardial disease advocates a more liberal application of EMB with immunohistochemical and viral genomic analysis in the evaluation of patients with suspected myocarditis. ${ }^{1}$

For a multitude of reasons, EMB is not routinely employed in the diagnosis of myocarditis. However, if EMB is performed in experienced centres, its major complication rate is $<1 \%$, which is similar to that of coronary angiography. ${ }^{61,62}$ Because the diagnostic yield of EMB is low in many instances, considerations for LV, biventricular, and image-guided biopsy techniques have been studied. The question of whether the location of biopsy sample might affect the accuracy of EMB-based diagnosis was reviewed in a study of 755 patients with clinically suspected myocarditis and/or cardiomyopathy of nonischaemic origin. ${ }^{62}$ Patients underwent selective LV EMB, selective right ventricular (RV) EMB, or biventricular EMB. CMR was also performed in the majority of these patients. The major complication rates were $0.64 \%$ for LV EMB and $0.82 \%$ for RV EMB. Diagnostic EMB results were achieved significantly more often in those patients who underwent biventricular EMBs (79.3\%) than in those who underwent either selective LV EMB or selective RV EMB (67.3\%; $P<0.001)$. The diagnostic utility of $\mathrm{LV}, \mathrm{RV}$, and biventricular EMB was also examined in 4,221 patients with unexplained dilated cardiomyopathy. ${ }^{63}$ Myocarditis was detected in $47 \%$ of LV EMB, $33 \%$ of RV EMB, and $49.6 \%$ of biventricular biopsies.

CMR is particularly useful in facilitating a guided approach by virtue of its utility in distinguishing between diseased and normal myocardium. The hypothesis that CMR might aid EMB-based diagnosis was tested when LGE-guided biopsy of the left and right ventricles was used in 32 patients with suspected myocarditis. ${ }^{64}$ LGEguided biopsy resulted in positive and negative predictive values for detecting myocarditis in $71 \%$ and $100 \%$ of patients, respectively. However, another study to assess whether the diagnostic yield of EMB might be improved with tissue taken from sites of LGE provided contrasting findings. For 116 patients who underwent biventricular EMBs (with biopsy-proven myocarditis) and a CMR study showing LGE, the distribution of LGE in relation to the EMB results was analysed. ${ }^{62}$ No significant differences were found in the number of diagnostic LV, RV, or biventricular EMBs when related to the site of LGE. Reasons for this finding include the fact that LGE is a nonspecific feature of myocardial injury that might be present in patients with both active and healed myocarditis. ${ }^{41}$ Alternative CMR sequences directed at active inflammation, such as the T2-weighted sequence, might prove to be a better guide for EMB, although this theory has yet to be studied. ${ }^{41}$

\section{Status of immunosuppression}

Therapy for myocarditis currently focuses largely on supportive care with attention to guideline-directed 
Table 1 | Prospective randomized, controlled trials of immunosuppression and immunomodulation in myocarditis

\begin{tabular}{|c|c|c|c|}
\hline Authors & Design & Primary end point & Results \\
\hline $\begin{array}{l}\text { Parrillo } \\
\text { et al. } 68\end{array}$ & $\begin{array}{l}\text { Randomized, controlled trial of } 102 \\
\text { patients with a history of idiopathic DCM } \\
\text { to compare prednisone with placebo }\end{array}$ & $\begin{array}{l}\text { Improved LVEF at } 3 \text { months } \\
\text { or reduced LV end-diastolic } \\
\text { dimensions }\end{array}$ & $\begin{array}{l}\text { Mean LVEF increased } 4.3 \pm 1.5 \% \text { in the } \\
\text { prednisone group compared with } \\
2.1 \pm 0.8 \% \text { in the control group }(P<0.054)\end{array}$ \\
\hline $\begin{array}{l}\text { Mason } \\
\text { et al. }{ }^{29}\end{array}$ & $\begin{array}{l}\text { Randomized, controlled trial of } 111 \\
\text { patients with biopsy-proven myocarditis } \\
\text { (unknown aetiology) to compare } \\
\text { prednisone plus ciclosporin or } \\
\text { azathioprine with conventional therapy }\end{array}$ & LVEF at 28 weeks & $\begin{array}{l}\text { No difference in LVEF or survival between } \\
\text { the two groups }(P=0.96)\end{array}$ \\
\hline $\begin{array}{l}\text { Wojnicz } \\
\text { et al. }{ }^{69}\end{array}$ & $\begin{array}{l}\text { Randomized, controlled trial of } 84 \text { patients } \\
\text { with inflammatory DCM (unknown } \\
\text { aetiology, increased HLA expression on } \\
\text { EMB) to compare prednisone plus } \\
\text { azathioprine with placebo }\end{array}$ & $\begin{array}{l}\text { A composite of death, } \\
\text { heart transplantation, } \\
\text { and hospital readmission } \\
\text { over } 2 \text { years }\end{array}$ & $\begin{array}{l}\text { No significant difference in primary end } \\
\text { point ( } 22.8 \% \text { for the immunosuppression } \\
\text { group vs } 20.5 \% \text { for the placebo group) }\end{array}$ \\
\hline $\begin{array}{l}\text { Frustaci } \\
\text { et al. }{ }^{70}\end{array}$ & $\begin{array}{l}\text { Randomized, controlled trial of } 85 \text { patients } \\
\text { with inflammatory, virus-negative DCM to } \\
\text { compare prednisone plus azathioprine } \\
\text { with placebo }\end{array}$ & LVEF at 6 months & $\begin{array}{l}\text { Significantly improved LVEF and } \\
\text { decreased LV dimensions in } \\
\text { immunosuppressive group }\end{array}$ \\
\hline $\begin{array}{l}\text { McNamara } \\
\text { et al. }{ }^{74}\end{array}$ & $\begin{array}{l}\text { Randomized, controlled trial of } 62 \text { patients } \\
\text { with recent-onset unexplained DCM } \\
\text { ( } \leq 6 \text { months) to compare IVIG with placebo }\end{array}$ & $\begin{array}{l}\text { Change in LVEF at } \\
6 \text { months and } 12 \text { months }\end{array}$ & $\begin{array}{l}\text { Both groups had similarly improved LVEF } \\
\text { at } 6 \text { months and } 12 \text { months }\end{array}$ \\
\hline $\begin{array}{l}\text { Gullestad } \\
\text { et } a .^{75}\end{array}$ & $\begin{array}{l}\text { Randomized, controlled trial of } 40 \text { patients } \\
\text { with chronic DCM to compare IVIG } \\
\text { with placebo }\end{array}$ & $\begin{array}{l}\text { Change in LVEF at } \\
6 \text { months }\end{array}$ & $\begin{array}{l}\text { Improved LVEF at } 6 \text { months in IVIG group } \\
\text { (LVEF increased from } 26 \pm 2 \% \text { to } 31 \pm 3 \% \text {, } \\
P<0.01 \text { ) compared with placebo } \\
\text { Marked increase in plasma levels of } \\
\text { anti-inflammatory mediators in IVIG group }\end{array}$ \\
\hline
\end{tabular}

treatment of heart failure and arrhythmia. ${ }^{1}$ Patients who present with haemodynamic collapse might require mechanical support, either as a bridge to transplantation or recovery. While NSAIDs and colchicine are indicated for the treatment of acute pericarditis, NSAIDs have been associated with increased mortality in animal models of myocarditis. ${ }^{65,66}$ As the pathogenesis of myocardial dysfunction apparent in myocarditis is a consequence of a maladaptive hyperimmune response triggered by viral infection, therapy directed towards modulating the immune response has been considered potentially beneficial. This hypothesis has been tested in both observational as well as controlled clinical trials. Although some investigators were optimistic after the results of several small observational studies, the high incidence of clinical improvement with standard heart failure treatment and substantial rate of spontaneous improvement in LV function substantiated the need for a control arm to evaluate treatment efficacy properly. ${ }^{67}$ Accordingly, we will focus on the large randomized, controlled clinical trials reporting on the use of immunosuppressive therapy (Table 1).

The first of these trials was designed to study the use of prednisone in patients with an unexplained dilated cardiomyopathy and the presence of inflammatory features confirmed by serum biomarkers, histopathology, or nuclear imaging. ${ }^{68}$ Reactive patients (defined as those who had fibroblastic or lymphocytic infiltration or immunoglobulin deposition on EMB) treated with prednisone were more likely than reactive controls to have $\geq 5 \%$ improvement in LV ejection fraction (LVEF) at 3 months. However, this improvement was not sustained at 6 months or 9 months. The Myocarditis Treatment Trial investigators randomly assigned 111 patients with biopsy-verified myocarditis, symptom duration of $<24$ months, and LVEF $\leq 35 \%$ to receive conventional therapy or immunosuppressive therapy in the form of prednisone and either azathioprine or ciclosporin. ${ }^{29}$ After 1 year of treatment, mortality and improvement in LV systolic function did not significantly differ between the two groups. LVEF improved by $10 \%$ in both cohorts. Notably, neither immunohistochemistry nor viral genomic analyses were performed on the biopsy specimens in this study, so patients with ongoing viral infection might have been treated with immunosuppressive therapy, thereby affecting the results. In another study, 84 patients with dilated cardiomyopathy, symptom duration of $>6$ months, and increased HLA expression on EMB (marker of persistent immune activation and chronic inflammation) were randomly assigned to receive placebo or immunsuppression in the form of prednisone and azathioprine. ${ }^{69}$ No significant differences were detected in the primary composite end point of death, heart transplantation, or hospital readmissions after 2 years between the two study groups. However, at 3 months and 2 years, LV systolic function and other echocardiographic indices had significantly improved with immunosuppression compared with placebo.

The presence of virus in biopsy specimens is relevant, given that one randomized immunosuppressive trial demonstrated improved LV systolic function and decreased LV dimensions at 6 months in patients with chronic, virus-negative myocarditis refractory to standard therapy. ${ }^{70}$ In this study, 85 patients with biopsy-proven 
Box 1 | Current recommendations for immunosuppressive therapy from the ESC

1. Immunosuppression should be started only after ruling out active infection on EMB by PCR

2. Based on experience with noncardiac autoimmune disease, consideration of immunosuppression in proven autoimmune (for example, infectionnegative) forms of myocarditis, should be made if no contraindications to immunosuppression are present, including giant-cell myocarditis, cardiac sarcoidosis, and myocarditis associated with known extracardiac autoimmune disease

3. Steroid therapy is indicated in cardiac sarcoidosis in the presence of ventricular dysfunction and/or arrhythmia and in some forms of infectionnegative eosinophilic or toxic myocarditis with heart failure and/or arrhythmia

4. Immunosuppression can be considered, on an individual basis, in infectionnegative lymphocytic myocarditis refractory to standard therapy in patients with no contraindications to immunosuppression

5. Follow-up EMB can be required to guide the intensity and the length of immunosuppression

Abbreviations: EMB, endomyocardial biopsy; ESC, European Society of Cardiology. Adapted from Caforio, A. L. P. et al. Current state of knowledge on aetiology, diagnosis, management, and therapy of myocarditis: a position statement from the European Society of Cardiology Working Group on Myocardial and Pericardial Diseases. Eur. Heart J. 34 (33), 2636-2648 @ (2013), with permission from Oxford University Press and the European Society of Cardiology.

virus-negative inflammatory cardiomyopathy were randomly assigned to placebo or to prednisone and azathioprine for 6 months. Echocardiographic features in $88 \%$ of patients who received immunosuppression significantly improved, whereas no patients in the control arm had any improvement in their LV systolic function. Notably, although the literature suggests that patients with viral myocarditis should not be treated with immunosuppression without biopsy-proven viral clearance, autoimmune myocarditis (including giant-cell myocarditis, sarcoidosis, lupus erythematosus, and eosinophilic myocarditis) is characterized by a profound response to glucocorticoids and immunosuppressive therapy. ${ }^{40,71}$ Current recommendations for immunosuppressive therapy are summarized in Box 1.

\section{Immunomodulatory therapy}

Intravenous immunoglobulin (IVIG) is a potent immunomodulatory therapy typically used in patients with systemic, antibody-mediated autoimmune disease, including giant-cell myocarditis. ${ }^{72,73}$ Thus far, the role of IVIG in viral myocarditis remains largely investigational. Its use was evaluated prospectively in a multicentre, double-blinded, randomized, controlled trial in 62 patients with recent-onset heart failure and dilated cardiomyopathy (defined as $\leq 6$ months). ${ }^{74}$ Biopsyproven myocarditis of unspecified aetiology was present in $16 \%$ of patients. No treatment benefit was observed, either in terms of mortality or improved LV systolic function at 6 months or 12 months. The potential benefit of IVIG was also studied in a prospective, double-blinded, randomized, placebo-controlled trial in 40 patients with chronic dilated cardiomyopathy. ${ }^{75} \mathrm{EMB}$ was not performed in these patients. IVIG was associated with significant improvement in LV systolic function (LVEF $26 \pm 2 \%$ to $31 \pm 3 \%, P<0.01)$ as well as a significant increase in plasma levels of anti-inflammatory mediators. Studies in the paediatric population have also shown that the use of high-dose IVIG for treatment of acute myocarditis is associated with improved recovery of LV function and an increased probability of survival during the first year after presentation. ${ }^{76}$ However, multicentre, placebo-controlled trials using IVIG in adult patients are still required to establish its efficacy in patients with biopsy-proven myocarditis.

As various autoantibodies targeting cardiac cell proteins have been detected in patients with myocarditis with a presumed pathogenic role, therapeutic strategies such as immunoadsorption of disease-causing antibodies have been suggested. ${ }^{1,77}$ The rationale for the application of immunoadsorption stems from its use in other autoimmune, antibody-mediated disorders. ${ }^{78,79}$ In small studies, immunoadsorption in patients with dilated cardiomyopathy has been shown to improve LV systolic function and levels of heart failure biomarkers, reduce myocardial inflammation, and ameliorate important haemodynamic parameters, including systemic vascular resistance and cardiac output indices. ${ }^{77,80-83}$ Although promising, current guidelines do not give recommendations for the use of immunoadsorption in patients with myocarditis, owing to the lack of large-scale, randomized, controlled trials to evaluate this treatment strategy. ${ }^{1}$ However, a large, prospective, multicentre, randomized, controlled trial on the therapeutic effect of immunoadsorption for patients with myocarditisrelated dilated cardiomyopathy is currently underway in Europe ${ }^{84}$ Lastly, despite a wealth of preclinical data indicating a potential therapeutic role for TNF antagonism, ${ }^{85}$ several clinical trials have failed to demonstrate a clinical benefit of using soluble TNF antagonists in patients with chronic heart failure; the data indicate the therapy could actually be harmful. ${ }^{85,86}$

\section{Antiviral therapy}

Currently, no approved pathogen-directed or antiviral therapies for patients with viral myocarditis exist. Treatment with aciclovir, ganciclovir, or valaciclovir for herpesvirus infection might be considered, although their efficacy has not been directly evaluated in patients with myocarditis. ${ }^{87}$ Vaccines could be a valuable option in the future, but have yet to be tested in myocarditis trials. ${ }^{88}$ In experimental animal studies, IFN- $\alpha$ and IFN- $\beta$ reduced myocardial viral replication and damage, with IFN- $\beta$ treatment more effective than IFN- $\alpha$ at complete elimination of cardiac viral load. ${ }^{88,89}$ Spontaneous and treatment-related viral clearance with IFN- $\beta$ has been associated with clinical and haemodynamic improvement in patients with myocarditis, whereas viral persistence remains a marker of poor outcomes as a result of progressive LV dysfunction. ${ }^{27,59,90}$ In a pilot study of 22 patients with persistent or progressive LV dysfunction, a 6 -month treatment course with IFN- $\beta$ effectively cleared enterovirus or adenovirus from the hearts of $100 \%$ of patients, with a resultant improvement in LV function in $68 \%$ of these patients. ${ }^{90}$ However, IFN- $\beta$ treatment has proven less effective in clearing parvovirus B19 infection. ${ }^{91}$ Again, large, randomized, placebo-controlled trials are required to establish the efficacy of antiviral therapy in patients with myocarditis. 


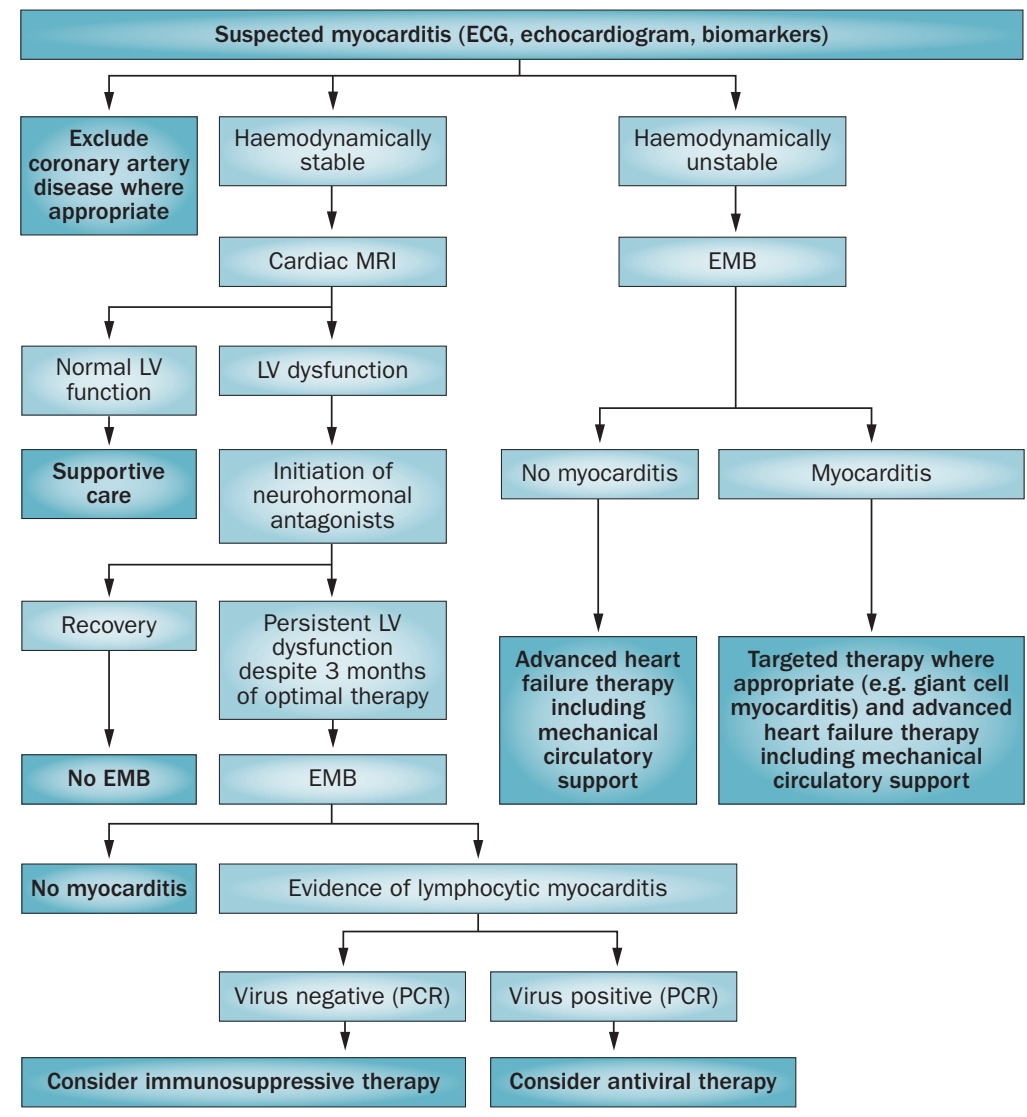

Figure 4 | Diagnostic and clinical algorithm for myocarditis. Comprehensive clinical practice guidelines specific to the treatment of myocarditis do not exist. We have proposed this algorithm for the diagnosis and treatment of patients with myocarditis. If any uncertainty exists with regard to the diagnosis, cardiac MRI is extremely valuable, owing to its high specificity and capacity to distinguish myocarditis from other myocardial disease processes. Abbreviations: ECG, electrocardiogram; EMB, endomyocardial biopsy; LV, left ventricular.

\section{Latest advances and future directions}

Although the pathogenesis of viral myocarditis has been described in animal models and is supported by pathological data from humans, whether the process is fully recapitulated in humans is unclear. Human mature cardiomyocytes can be procured only through invasive myocardial biopsy and are not easily cultured in vitro, which poses a challenge to studying the course of infection in human myocardium. However, advances in somatic cell reprogramming technology have enabled the generation of induced pluripotent stem cells (iPSCs) from human dermal fibroblasts, which can then be readily differentiated into cardiomyocytes. Such iPSC-derived cardiomyocytes (iPSC-CMs) have already been used to study human cardiovascular disease. ${ }^{92}$ An in vitro model of human viral myocarditis has been developed by infecting human iPSC-CMs with coxsackievirus. ${ }^{93}$ Infected cells harboured deleterious changes in myocyte structure and function and responded variably to several antiviral compounds. This iPSC-CM model is an exciting platform for studying disease mechanisms and for high-throughput screening of potential therapeutics.
In the early 1980s, it was recognized that patients with viral myocarditis had circulating antisarcolemmal and antiendothelial antibodies that induced complement-mediated cytotoxic reactions. ${ }^{94,95}$ More than 3 decades later, the underpinnings of myocardial response to viral exposure remain elusive. If more than half the population is seropositive for coxsackievirus, ${ }^{96}$ why is viral myocarditis such a relatively rare phenomenon? Indeed, $76 \%$ of patients who become infected with coxsackievirus have no apparent clinical manifestations. ${ }^{97}$ Therefore, a set of human host genetic factors might exist that confer predilection to, or protection from, myocardial injury as suggested by animal models. Indeed, modification of genetically determined immune responses in some mouse strains alters viral elimination from the heart during the acute stages of infection, and such strains undergo substantial cardiac remodelling leading to a chronic form of myocarditis. ${ }^{98}$ Thus, identifying the molecular basis for differences in susceptibility between individuals could open new therapeutic windows for preventing or treating viral myocarditis.

In practice, physicians rely on an amalgamation of clinical features, laboratory analyses, and imaging data to evaluate and care for patients with suspected myocarditis. Without formal guidelines, no blueprint exists to steer practitioners towards a uniform pathway for evaluation, diagnosis, and treatment of this disease. We suggest an algorithm delineating a step-wise approach to the diagnosis and treatment of patients with suspected myocarditis (Figure 4). We propose that EMB be performed on individuals presenting with sudden and severe symptoms or persistent LV dysfunction after initiation of neurohormonal therapy. This strategy emanates from our own clinical experience in conjunction with the AHA/ACC/ESC guidelines on the role of EMB in the management of cardiovascular disease. ${ }^{48}$

\section{Conclusions}

Myocarditis typically results from infection by a cardiotropic virus followed by active inflammatory destruction of the myocardium. As a disease entity, characterization of myocarditis has been hampered by its heterogeneous clinical presentations and diverse aetiologies. CMR is a helpful adjunct in establishing the diagnosis, although EMB remains the current gold standard. Targeted sample collection guided by CMR and more sophisticated analysis of biopsy specimens with detection of viral genomes and immunohistochemical evaluation could lead to expanded indications for pursuing EMB (Figure 4). Treatment of myocarditis remains largely supportive. Immunosuppressive therapy can be beneficial in patients with giant-cell myocarditis and sarcoidosis; however, its role in chronic, virus-negative myocarditis, although promising, requires further elucidation. The use of immunomodulatory and antiviral therapy remains largely investigational at this time. Large, randomized, controlled trials are needed to determine their role in the treatment of inflammatory heart disease. 
1. Caforio, A. L. et al. Current state of knowledge on aetiology, diagnosis, management, and therapy of myocarditis: a position statement of the European Society of Cardiology Working Group on Myocardial and Pericardial Diseases. Eur. Heart J. 34, 2636-2648 (2013).

2. Richardson, P. et al. Report of the 1995 World Health Organization/International Society and Federation of Cardiology Task Force on the Definition and Classification of Cardiomyopathies. Circulation 93, 841-842 (1996).

3. Breinholt, J. P. et al. Viral epidemiologic shift in inflammatory heart disease: the increasing involvement of parvovirus B19 in the myocardium of pediatric cardiac transplant patients. J. Heart Lung Transplant. 29, 739-746 (2010).

4. Schultz, J. C., Hilliard, A. A., Cooper, L. T. Jr \& Rihal, C. S. Diagnosis and treatment of viral myocarditis. Mayo Clin. Proc. 84, 1001-1009 (2009).

5. Magnani, J. W. \& Dec, G. W. Myocarditis: current trends in diagnosis and treatment. Circulation 113, 876-890 (2006).

6. Towbin, J. A. et al. Incidence, causes, and outcomes of dilated cardiomyopathy in children. JAMA 296, 1867-1876 (2006).

7. Doolan, A., Langlois, N. \& Semsarian, C. Causes of sudden cardiac death in young Australians. Med. J. Aust. 180, 110-112 (2004).

8. Fabre, A. \& Sheppard, M. N. Sudden adult death syndrome and other non-ischaemic causes of sudden cardiac death. Heart 92, 316-320 (2006).

9. Felker, G. M. et al. The spectrum of dilated cardiomyopathy. The Johns Hopkins experience with 1,278 patients. Medicine (Baltimore) 78, 270-283 (1999).

10. Maron, B. J. et al. Contemporary definitions and classification of the cardiomyopathies: an American Heart Association Scientific Statement from the Council on Clinical Cardiology, Heart Failure and Transplantation Committee; Quality of Care and Outcomes Research and Functional Genomics and Translational Biology Interdisciplinary Working Groups; and Council on Epidemiology and Prevention. Circulation 113, 1807-1816 (2006).

11. Aretz, H. T. et al. Myocarditis. A histopathologic definition and classification. Am. J. Cardiovasc. Pathol. 1, 3-14 (1987).

12. Baughman, K. L. Diagnosis of myocarditis: death of Dallas criteria. Circulation 113, 593-595 (2006).

13. Wu, L. A., Lapeyre, A. C. 3rd \& Cooper, L. T. Current role of endomyocardial biopsy in the management of dilated cardiomyopathy and myocarditis. Mayo Clin. Proc. 76, 1030-1038 (2001).

14. Hauck, A. J., Kearney, D. L. \& Edwards, W. D. Evaluation of postmortem endomyocardial biopsy specimens from 38 patients with lymphocytic myocarditis: implications for role of sampling error. Mayo Clin. Proc. 64, 1235-1245 (1989).

15. Kindermann, I. et al. Update on myocarditis. J. Am. Coll. Cardiol. 59, 779-792 (2012).

16. Fairley, C. K., Ryan, M., Wall, P. G. \& Weinberg, J. The organisms reported to cause infective myocarditis and pericarditis in England and Wales. J. Infect. 32, 223-225 (1996).

17. Kawai, C. Idiopathic cardiomyopathy. A study on the infectious-immune theory as a cause of the disease. Jpn. Circ. J. 35, 765-770 (1971).

18. Freimuth, P., Philipson, L. \& Carson, SD. The coxsackievirus and adenovirus receptor. Curr. Top. Microbiol. Immunol. 323, 67-87 (2008).
19. Pinkert, S. et al. Prevention of cardiac dysfunction in acute coxsackievirus B3 cardiomyopathy by inducible expression of a soluble coxsackievirus-adenovirus receptor. Circulation 120, 2358-2366 (2009).

20. Blailock, Z. R., Rabin, E. R. \& Melnick, J. L. Adenovirus myocarditis in mice. An electron microscopic study. Exp. Mol. Pathol. 9, 84-96 (1968).

21. Shi, Y. et al. Cardiac deletion of the coxsackievirus-adenovirus receptor abolishes coxsackievirus B3 infection and prevents myocarditis in vivo. J. Am. Coll. Cardiol. 53 1219-1226 (2009).

22. Kandolf, R., Ameis, D., Kirschner, P., Canu, A. \& Hofschneider, P. H. In situ detection of enteroviral genomes in myocardial cells by nucleic acid hybridization: an approach to the diagnosis of viral heart disease. Proc. Natl Acad. Sci. USA 84, 6272-6276 (1987).

23. Badorff, C. et al. Enteroviral protease $2 A$ cleaves dystrophin: evidence of cytoskeletal disruption in an acquired cardiomyopathy. Nat. Med. 5, 320-326 (1999).

24. Bültmann, B. D. et al. Fatal parvovirus B19associated myocarditis clinically mimicking ischemic heart disease: an endothelial cellmediated disease. Hum. Pathol. 34, 92-95 (2003).

25. Bock, C. T., Klingel, K. \& Kandolf, R. Human parvovirus B19-associated myocarditis. N. Engl. J. Med. 362, 1248-1249 (2010).

26. Kühl, U. et al. High prevalence of viral genomes and multiple viral infections in the myocardium of adults with "idiopathic" left ventricular dysfunction. Circulation 111, 887-893 (2005).

27. Kühl, U. et al. Viral persistence in the myocardium is associated with progressive cardiac dysfunction. Circulation 112, 1965-1970 (2005).

28. Caforio, A. L. et al. A prospective study of biopsy-proven myocarditis: prognostic relevance of clinical and aetiopathogenetic features at diagnosis. Eur. Heart J. 28, 1326-1333 (2007).

29. Mason, J. W. et al. A clinical trial of immunosuppressive therapy for myocarditis. The Myocarditis Treatment Trial Investigators. N. Engl. J. Med. 333, 269-275 (1995).

30. Smith, S. C., Ladenson, J. H., Mason, J. W. \& Jaffe, A. S. Elevations of cardiac troponin I associated with myocarditis. Experimental and clinical correlates. Circulation 95, 163-168 (1997).

31. Mahfoud, F. et al. Virus serology in patients with suspected myocarditis: utility or futility? Eur. Heart J. 32, 897-903 (2011).

32. Dec, G. W. Jr. et al. Viral myocarditis mimicking acute myocardial infarction. J. Am. Coll. Cardiol. 20, 85-89 (1992).

33. Angelini, A. et al. Myocarditis mimicking acute myocardial infarction: role of endomyocardial biopsy in the differential diagnosis. Heart 84, 245-250 (2000).

34. Friedrich, M. G. et al. Contrast media-enhanced magnetic resonance imaging visualizes myocardial changes in the course of viral myocarditis. Circulation 97, 1802-1809 (1998).

35. Felker, G. M. et al. Echocardiographic findings in fulminant and acute myocarditis. J. Am. Coll. Cardiol. 36, 227-232 (2000).

36. Herskowitz, A. et al. Demographic features and prevalence of idiopathic myocarditis in patients undergoing endomyocardial biopsy. Am. J. Cardiol. 71, 982-986 (1993).

37. Kindermann, I. et al. Predictors of outcome in patients with suspected myocarditis. Circulation 118, 639-648 (2008).
38. McCarthy, R. E. 3rd et al. Long-term outcome of fulminant myocarditis as compared with acute (nonfulminant) myocarditis. N. Engl. J. Med. 342, 690-695 (2000).

39. Anzini, M. et al. Long-term evolution and prognostic stratification of biopsy-proven active myocarditis. Circulation 128, 2384-2394 (2013).

40. Cooper, L. T. Jr, Berry, G. J. \& Shabetai, R. Idiopathic giant-cell myocarditis-natural history and treatment. Multicenter Giant Cell Myocarditis Study Group Investigators. N. Engl. J. Med. 336, 1860-1866 (1997).

41. Yilmaz, A. et al. Role of cardiovascular magnetic resonance imaging (CMR) in the diagnosis of acute and chronic myocarditis. Heart Fail. Rev. 18, 747-760 (2013).

42. Friedrich, M. G. et al. Cardiovascular magnetic resonance in myocarditis: A JACC White Paper. J. Am. Coll. Cardiol. 53, 1475-1487 (2009).

43. Abdel-Aty, H. et al. Diagnostic performance of cardiovascular magnetic resonance in patients with suspected acute myocarditis: comparison of different approaches. J. Am. Coll. Cardiol. 45 1815-1822 (2005).

44. Mahrholdt, H. et al. Presentation, patterns of myocardial damage, and clinical course of viral myocarditis. Circulation 114, 1581-1590 (2006).

45. Grün, S. et al. Long-term follow-up of biopsyproven viral myocarditis: predictors of mortality and incomplete recovery. J. Am. Coll. Cardiol. 59, 1604-1615 (2012).

46. Baccouche, H. et al. Diagnostic synergy of noninvasive cardiovascular magnetic resonance and invasive endomyocardial biopsy in troponinpositive patients without coronary artery disease. Eur. Heart J. 30, 2869-2879 (2009).

47. Gutberlet, M. et al. Suspected chronic myocarditis at cardiac MR: diagnostic accuracy and association with immunohistologically detected inflammation and viral persistence. Radiology 246, 401-409 (2008).

48. Cooper, L. T. et al. The role of endomyocardial biopsy in the management of cardiovascular disease: a scientific statement from the American Heart Association, the American College of Cardiology, and the European Society of Cardiology. Endorsed by the Heart Failure Society of America and the Heart Failure Association of the European Society of Cardiology. J. Am. Coll. Cardiol. 50, 1914-1931 (2007).

49. Jacoby, C. et al. Visualization of immune cell infiltration in experimental viral myocarditis by ${ }^{19} \mathrm{~F}$ MRI in vivo. MAGMA 27, 101-106 (2014).

50. Bates, D., Abraham, S., Campbell, M., Zehbe, I. $\&$ Curiel, L. Development and characterization of an antibody-labeled super-paramagnetic iron oxide contrast agent targeting prostate cancer cells for magnetic resonance imaging. PLOS ONE 9, e97220 (2014)

51. Shahbazi-Gahrouei, D., Williams, M., Rizvi, S. \& Allen, B. J. In vivo studies of Gd-DTPAmonoclonal antibody and gd-porphyrins: potential magnetic resonance imaging contrast agents for melanoma. J. Magn. Reson. Imaging 14, 169-174 (2001).

52. Jamar, F. et al. EANM/SNMMI guideline for ${ }^{18} \mathrm{~F}-\mathrm{FDG}$ use in inflammation and infection. J. Nucl. Med. 54, 647-658 (2013).

53. Ohira, H. et al. Myocardial imaging with ${ }^{18} \mathrm{~F}$-fluoro-2-deoxyglucose positron emission tomography and magnetic resonance imaging in sarcoidosis. Eur. J. Nucl. Med. Mol. Imaging 35 , 933-941 (2008).

54. Brudin, L. H. et al. Fluorine-18 deoxyglucose uptake in sarcoidosis measured with positron 
emission tomography. Eur. J. Nucl. Med. 21, 297-305 (1994).

55. Blankstein, R. et al. Cardiac positron emission tomography enhances prognostic assessments of patients with suspected cardiac sarcoidosis. J. Am. Coll. Cardiol. 63, 329-336 (2014).

56. Klingel, K. et al. Pathogenesis of murine enterovirus myocarditis: virus dissemination and immune cell targets. J. Virol. 70, 8888-8895 (1996).

57. Calabrese, F. \& Thiene, G. Myocarditis and inflammatory cardiomyopathy: microbiological and molecular biological aspects. Cardiovasc. Res. 60, 11-25 (2003).

58. Dennert, R., Crijns, H. J. \& Heymans, S. Acute viral myocarditis. Eur. Heart J. 29, 2073-2082 (2008).

59. Why, H. J. et al. Clinical and prognostic significance of detection of enteroviral RNA in the myocardium of patients with myocarditis or dilated cardiomyopathy. Circulation 89, 25822589 (1994).

60. Leone, O. et al. 2011 consensus statement on endomyocardial biopsy from the Association for European Cardiovascular Pathology and the Society for Cardiovascular Pathology. Cardiovasc. Pathol. 21, 245-274 (2012).

61. Holzmann, M. et al. Complication rate of right ventricular endomyocardial biopsy via the femoral approach: a retrospective and prospective study analyzing 3048 diagnostic procedures over an 11-year period. Circulation 118, 1722-1728 (2008).

62. Yilmaz, A. et al. Comparative evaluation of left and right ventricular endomyocardial biopsy: differences in complication rate and diagnostic performance. Circulation 122, 900-909 (2010).

63. Chimenti, C. \& Frustaci, A. Contribution and risks of left ventricular endomyocardial biopsy in patients with cardiomyopathies: a retrospective study over a 28-year period. Circulation 128, 1531-1541 (2013).

64. Mahrholdt, H. et al. Cardiovascular magnetic resonance assessment of human myocarditis: a comparison to histology and molecular pathology. Circulation 109, 1250-1258 (2004).

65. Feldman, A. M. \& McNamara, D. Myocarditis. N. Engl. J. Med. 343, 1388-1398 (2000).

66. Costanzo-Nordin, M. R., Reap, E. A., O'Connell, J. B., Robinson, J. A. \& Scanlon, P. J. A nonsteroid anti-inflammatory drug exacerbates Coxsackie B3 murine myocarditis. J. Am. Coll. Cardiol. 6, 1078-1082 (1985).

67. Hobbs, R. E. et al. Lymphocytic myocarditis and dilated cardiomyopathy: treatment with immunosuppressive agents. Cleve. Clin. J. Med. 56, 628-635 (1989).

68. Parrillo, J. E. et al. A prospective, randomized, controlled trial of prednisone for dilated cardiomyopathy. N. Engl. J. Med. 321, 1061-1068 (1989).

69. Wojnicz, R. et al. Randomized, placebo-controlled study for immunosuppressive treatment of inflammatory dilated cardiomyopathy: two-year follow-up results. Circulation 104, 39-45 (2001).

70. Frustaci, A., Russo, M. A. \& Chimenti, C. Randomized study on the efficacy of immunosuppressive therapy in patients with virus-negative inflammatory cardiomyopathy: the TIMIC study. Eur. Heart J. 30, 1995-2002 (2009).

71. Pulerwitz, T. C. et al. Mortality in primary and secondary myocarditis. Am. Heart J. 147, 746-750 (2004).

72. Orange, J. S. et al. Use of intravenous immunoglobulin in human disease: a review of evidence by members of the Primary Immunodeficiency Committee of the American Academy of Allergy, Asthma and Immunology. J. Allergy Clin. Immunol. 117, S525-S553 (2006).

73. Menghini, V. V. et al. Combined immunosuppression for the treatment of idiopathic giant cell myocarditis. Mayo Clin. Proc. 74, 1221-1226 (1999).

74. McNamara, D. M. et al. Controlled trial of intravenous immune globulin in recent-onset dilated cardiomyopathy. Circulation 103, 2254-2259 (2001).

75. Gullestad, L. et al. Immunomodulating therapy with intravenous immunoglobulin in patients with chronic heart failure. Circulation 103, 220-225 (2001).

76. Drucker, N. A. et al. $\gamma$-globulin treatment of acute myocarditis in the pediatric population. Circulation 89, 252-257 (1994).

77. Felix, S. B. et al. Removal of cardiodepressant antibodies in dilated cardiomyopathy by immunoadsorption. J. Am. Coll. Cardiol. 39, 646-652 (2002).

78. Bygren, P. et al. Goodpasture's syndrome treated with staphylococcal protein A immunoadsorption. Lancet 2, 1295-1296 (1985).

79. Palmer, A., Gjorstrup, P., Severn, A., Welsh, K. $\&$ Taube, D. Treatment of systemic lupus erythematosus by extracorporeal immunoadsorption. Lancet 2, 272 (1988).

80. Mobini, R. et al. Hemodynamic improvement and removal of autoantibodies against $\beta_{1}$-adrenergic receptor by immunoadsorption therapy in dilated cardiomyopathy. J. Autoimmun. 20, 345-350 (2003).

81. Herda, L. R. et al. Effects of immunoadsorption and subsequent immunoglobulin G substitution on cardiopulmonary exercise capacity in patients with dilated cardiomyopathy. Am. Heart J. 159, 809-816 (2010).

82. Felix, S. B. et al. Hemodynamic effects of immunoadsorption and subsequent immunoglobulin substitution in dilated cardiomyopathy: three-month results from a randomized study. J. Am. Coll. Cardiol. 35 1590-1598 (2000).

83. Doesch, A. O. et al. Effects of protein A immunoadsorption in patients with advanced chronic dilated cardiomyopathy. J. Clin. Apher. 24, 141-149 (2009).

84. US National Library of Medicine. ClinicalTrials.gov [online], https://clinicaltrials.gov/ct2/show/ NCT00558584 (2015).

85. Mann, D. L. et al. Targeted anticytokine therapy in patients with chronic heart failure: results of the Randomized Etanercept Worldwide Evaluation (RENEWAL). Circulation 109, 1594-1602 (2004).
86. Chung, E. S., Packer, M., Lo, K. H., Fasanmade, A. A. \& Willerson, J. T. Randomized, double-blind, placebo-controlled, pilot trial of infliximab, a chimeric monoclonal antibody to tumor necrosis factor- $\alpha$, in patients with moderate-to-severe heart failure: results of the anti-TNF Therapy Against Congestive Heart Failure (ATTACH) trial. Circulation 107, 3133-3140 (2003).

87. Krueger, G. R. \& Ablashi, D. V. Human herpesvirus-6: a short review of its biologica behavior. Intervirology 46, 257-269 (2003).

88. Martino, T. A., Liu, P. \& Sole, M. J. Viral infection and the pathogenesis of dilated cardiomyopathy. Circ. Res. 74, 182-188 (1994).

89. Wang, Y. X. et al. Antiviral and myocyte protective effects of murine interferon- $\beta$ and $-\alpha_{2}$ in coxsackievirus B3-induced myocarditis and epicarditis in Balb/c mice. Am J. Physiol. Heart Circ. Physiol. 293, H69-H76 (2007).

90. Kühl, U. et al. Interferon- $\beta$ treatment eliminates cardiotropic viruses and improves left ventricular function in patients with myocardial persistence of viral genomes and left ventricular dysfunction. Circulation 107, 2793-2798 (2003).

91. Schultheiss, H. et al. The effect of subcutaneous treatment with interferon- $\beta$ - $1 \mathrm{~b}$ over 24 weeks on safety, virus elimination and clinical outcome in patients with chronic viral cardiomyopathy [abstract]. Circulation 118, 3322 (2008)

92. Carvajal-Vergara, X. et al. Patient-specific induced pluripotent stem-cell-derived models of LEOPARD syndrome. Nature $465,808-812$ (2010).

93. Sharma, A. et al. Human induced pluripotent stem cell-derived cardiomyocytes as an in vitro model for coxsackievirus B3-induced myocarditis and antiviral drug screening platform. Circ. Res. 115, 556-566 (2014).

94. Maisch, B., Berg, P. A. \& Kochsiek, K. Autoantibodies and serum inhibition factors (sif) in patients with myocarditis. Klin. Wochenschr. 58, 219-225 (1980).

95. Maisch, B., Trostel-Soeder, R., Stechemesser, E. Berg, P. A. \& Kochsiek, K. Diagnostic relevance of humoral and cell-mediated immune reactions in patients with acute viral myocarditis. Clin. Exp. Immunol. 48, 533-545 (1982).

96. Minor, P. in Topley and Wilson's Microbiology and Microbial Infections 9th edn Vol. 1 Ch. 25 (eds Mahy, B. W. J. \& Collier, L.) 485-510 (Oxford University Press, 1998).

97. Kim, K. S., Hufnagel, G., Chapman, N. M. $\&$ Tracy, S. The group B coxsackieviruses and myocarditis. Rev. Med. Virol. 11, 355-368 (2001).

98. Szalay, G. et al. Osteopontin: a fibrosis-related marker molecule in cardiac remodeling of enterovirus myocarditis in the susceptible host. Circ. Res. 104, 851-859 (2009).

\section{Author contributions}

A.P. and A.R.K. researched data for the article. All authors substantially contributed to discussion of content, wrote the article, and reviewed/edited the manuscript before submission. 